\title{
Dynamic Analysis of Honeycomb Sandwich Beam with Multiple Debonds
}

\author{
B. Saraswathy, ${ }^{1}$ R. Ramesh Kumar, ${ }^{2}$ and Lalu Mangal ${ }^{1}$ \\ ${ }^{1}$ Department of Civil Engineering, TKM College of Engineering, Kerala, Kollam 691005, India \\ ${ }^{2}$ Honeycomb Production and Development Division, Vikram Sarabhai Space Centre, Kerala, Thiruvananthapuram 695022 , India
}

Correspondence should be addressed to B. Saraswathy, bsatkm@sify.com

Received 24 August 2011; Accepted 10 October 2011

Academic Editors: K. Ismail, N. Moes, and D. Zhou

Copyright ( 2012 B. Saraswathy et al. This is an open access article distributed under the Creative Commons Attribution License, which permits unrestricted use, distribution, and reproduction in any medium, provided the original work is properly cited.

\begin{abstract}
Analytical formulation for the evaluation of frequency of CFRP sandwich beam with debond, following the split beam theory, generally underestimates the stiffness, as the contact between the honeycomb core and the skin during vibration is not considered in the region of debond. The validation of the present analytical solution for multiple-debond size is established through 3D finite element analysis, wherein geometry of honeycomb core is modeled as it is, with contact element introduced in the debond region. Nonlinear transient analysis is followed by fast Fourier transform analysis to obtain the frequency response functions. Frequencies are obtained for two types of model having single debond and double debond, at different spacing between them, with debond size up to $40 \%$ of beam length. The analytical solution is validated for a debond length of $15 \%$ of the beam length, and with the presence of two debonds of same size, the reduction in frequency with respect to that of an intact beam is the same as that of a single-debond case, when the debonds are well separated by three times the size of debond. It is also observed that a single long debond can result in significant reduction in the frequencies of the beam than multiple debond of comparable length.
\end{abstract}

\section{Introduction}

Sandwich construction is formed by bonding two thin facings to a thick core and has very high strength and stiffness properties achieved by increasing the thickness of the core without any weight penalty. The manufacturing defects like incomplete wetting or entrapped air pockets into resin-dominant layer can result in nonuniform adhesion between the face sheets and the core or skin-to-core debond. In-service circumstances such as low velocity impact by foreign objects or accidental tool drops during maintenance operations can lead to local debond. Overloading and elevated temperature regime may also induce debond at the weakest point of skin-to-core interface. During service, the debond may propagate and trigger new damage modes such as face sheet wrinkling, dimpling, and core shear cracks. As the structures using sandwich materials require extremely high level of reliability, debond must be detected immediately after its occurrence to ensure safety and the durability of the structures.

In the author's recent analytical study on vibration characteristics of sandwich beams with debond, the well-known split beam theory was modified considering the core stiffness of sandwich beam and accordingly the equations of motion were derived [1]. In the case of an aluminium skinned cantilever sandwich beam with debond, the analytical results showed good agreement with test data up to debond of size $12 \%$ of beam length. It was concluded that the analytical model underestimates the frequencies for larger debond lengths due to the noninclusion of the support offered by the core in the debond region during vibration. In the absence of more test data, to validate the analytical solution, finite element analysis using contact elements in the debond region is used in the current work.

Goswami and Becker used the finite element method to study the cause and the effects of debonding phenomena in between the face sheet and the core of a sandwich plate under in-plane loading [2]. A "unit cell" approach has been followed throughout the study. Waldron et al. employed finite element method combined with operational deflection shapes (ODSs) for damage identification [3]. The actual vibration displacement or velocity pattern of a structure that is vibrating in the steady-state condition due to a specific structural loading is noted. The ODSs are represented as 


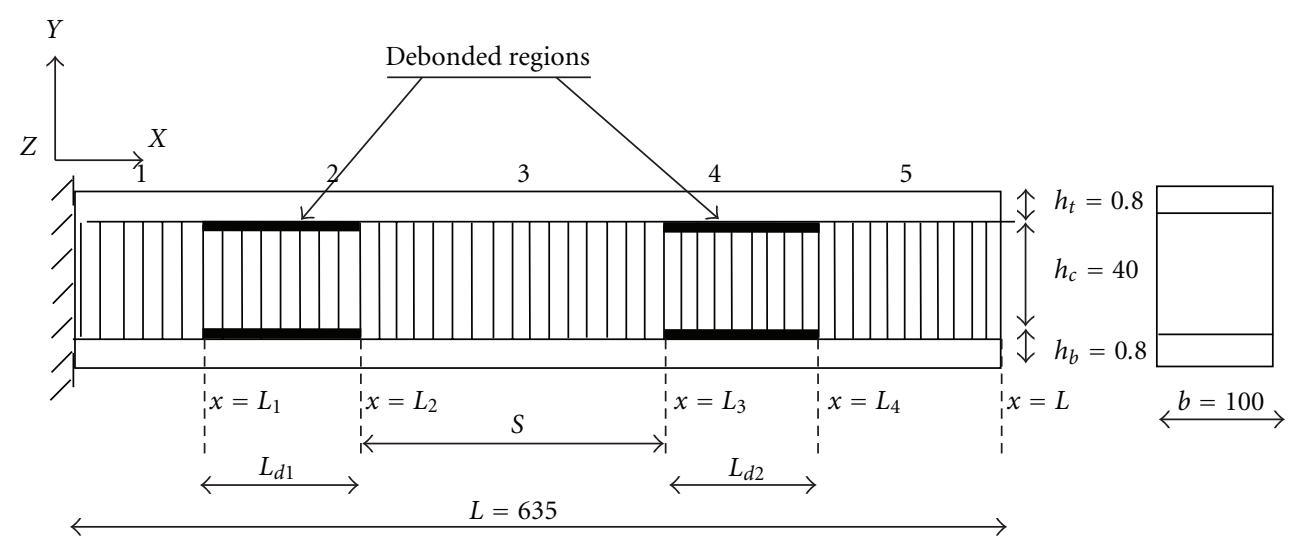

FIGURE 1: Schematic representation of the beam with debond.

summations of scaled mode shapes, and it is demonstrated through finite-element simulations how the vibration excitation parameters can be chosen to aid in identifying structural damages. The layerwise model for modeling composite laminate plates with embedded delaminations was used by Kim et al. [4]. To model delamination, the delaminated element with additional nodal unknowns was developed in their study. Virtual spring elements were utilized by Yam et al. to simulate the interface between upper and lower sublaminates in the delaminated region of laminated composite plates [5]. Cecchini examined various existing NDE techniques to assess damage in sandwich composites structures including vibration modal analysis, transient thermal response, and acoustic emission [6]. An experimentally validated neural network damage detection system for location and severity predictions of damage in sandwich composite structures was developed. Lestari and Qiao conducted a combined analytical and experimental study of dynamic characteristics of honeycomb composite sandwich structures in bridge systems [7]. They have proposed a relatively simple and reliable dynamic experimental procedure to estimate the beam bending and transverse shear stiffness. Burlayenko and Sadowski carried out analysis of structural performance of sandwich plate with hollow and foam-filled honeycomb cores using the finite element code ABAQUS [8]. The displacement-based homogeneous technique using FEM is applied to evaluate the effective elastic properties of hexagonal honeycomb core for both the cases. The structural benefits of foam-filled sandwich plates are discussed by comparing the results of load carrying capacities and natural frequency values. Burlayenko and Sadowski adopted the finite element code ABAQUS to calculate the modal parameters of damaged sandwich plates containing a debonding zone. The finite elements such as six- and eight-noded general-purpose shell elements and six- and eight-nodded with incompatible mode linear solid elements were applied to discretize the skins and the core, respectively. 3D spring elements were introduced between the double nodes in the debonded area [9].

The aim of the present study is to find out the limit of debond length up to which the analytical prediction of frequency is valid for the case of a CFRP sandwich beam with
TABLE 1: Properties of materials used.

\begin{tabular}{ll}
\hline Carbon-epoxy skin & Aluminium honeycomb core \\
\hline$E_{x x}=294.3 \mathrm{GPa}$, & Plate shear modulus \\
$E_{y y}=E_{z z}=5.957 \mathrm{GPa}$ & L-direction $=220.64 \mathrm{MPa}$ \\
& $W$-direction $=103.42 \mathrm{MPa}$ \\
$G_{x y}=G_{x z}=4.896 \mathrm{GPa}$ & Cell size $=6 \mathrm{~mm}$ \\
& (inscribed circle diameter) \\
$v=0.346$, & Cell wall thickness $=1 / 1000$ of an inch \\
$\rho=1700 \mathrm{~kg} / \mathrm{m}^{3}$ & $(.00254 \mathrm{~mm}), \rho=2.3 \mathrm{pcf}\left(33 \mathrm{~kg} / \mathrm{m}^{3}\right)$ \\
\hline
\end{tabular}

multiple debonds. For this purpose, finite element analysis is carried out and the results are compared with analytical solution for multiple debond of different lengths and spacing between them. In the numerical analysis using finite element method, contact elements are introduced between the skin and core in the debond region and nonlinear transient analysis is performed and frequency response functions are obtained through fast Fourier transform analysis.

\section{Details of the Model}

Honeycomb sandwich beams with laminated carbon-epoxy (CFRP) skins are considered in the present study. All the beams are of size $635 \times 100 \mathrm{~mm}$. The metallic honeycomb core is of aluminium alloy AA 5056 with core height $40 \mathrm{~mm}$ and the eight layered CFRP skins are of thickness $0.8 \mathrm{~mm}$, each layer $0.1 \mathrm{~mm}$ thick with lay up sequence $\left(0^{\circ} / 45^{\circ} /-45^{\circ} / 90^{\circ} / 90^{\circ} /-45^{\circ} / 45^{\circ} / 0^{\circ}\right)$. Sandwich beams without debond, with single debond, and with two debonds at different spacing between them are considered in the present analysis. Figure 1 gives the details of the debonded beam and material properties of the CFRP skin and core are provided in Table 1.

\section{Analytical Formulation}

Figure 1 represents a honeycomb sandwich beam having debond at two locations. The debonded sandwich beam is analysed as five-beam segments, the debond region, $i=2,4$, 


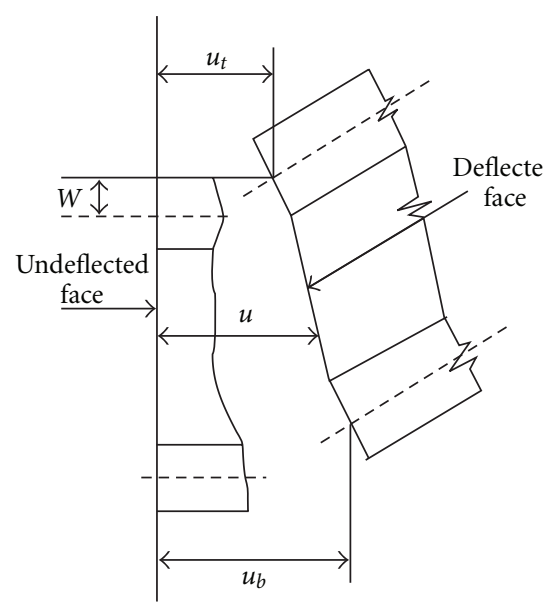

(a)

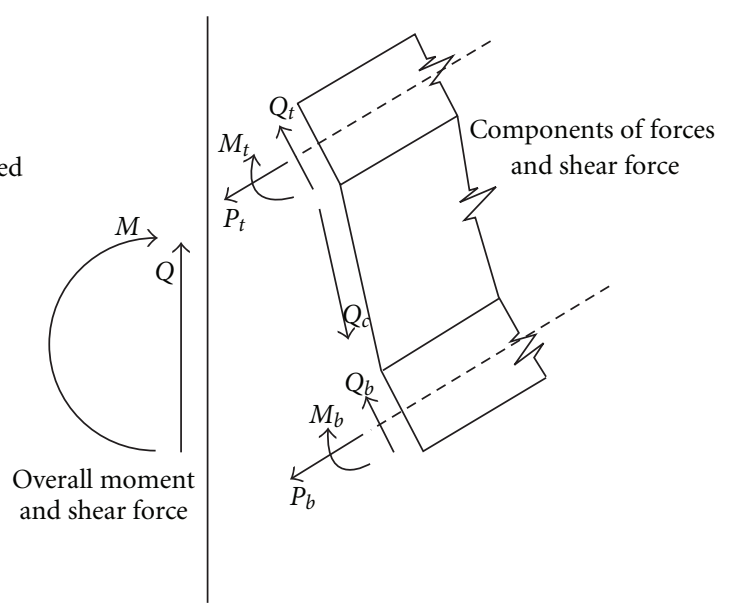

(b)

Figure 2: (a) Displacements of the sandwich beam. (b) Forces and moments in the sandwich beam.

and the other adjacent intact regions, denoted by $i=1,3,5$. The thickness of each of the top and bottom faces is $h_{f}$ and that of the core is $h_{c}$ and $d$ is the centre-to-centre distance between the top and bottom faces. The top and bottom faces possess axial and bending rigidity, whereas the core is assumed to possess only shear rigidity. Transverse flexural stresses in both core and face plates are also neglected so that the transverse displacements $w_{i}$ on a cross-section of the beam are equal.

3.1. Formulation for the Intact Region of the Beam. The centre line displacements of the top and bottom layers in the longitudinal direction are $u_{t}$ and $u_{b}$ as shown in Figure 2(a):

$$
u_{t}=-u_{b}
$$

The shear strain $\gamma$ in the core is given by

$$
\begin{gathered}
\gamma=\frac{\partial w}{\partial x}+\frac{\partial u}{\partial z}=\frac{d}{h_{c}} \frac{\partial w}{\partial x}+\frac{u_{t}-u_{b}}{h_{c}} . \\
d=h_{c}+\frac{\left(h_{t}+h_{b}\right)}{2} .
\end{gathered}
$$

The shear stress in the core $\tau=G_{c} \gamma$.

The shear force of the core $Q_{c}=-\tau b d$.

The total shear force, $Q$, across the thickness of a sandwich beam consists of $Q_{t}$ and $Q_{b}$ for the top and bottom face plates and also of a shear force $Q_{c}$ associated with the shear stress of the honeycomb core as given in Figure 2(b):

$$
\begin{gathered}
Q=D_{f} \frac{\partial^{3} w}{\partial x^{3}}-G_{c} b d\left(\frac{d}{h_{c}} \frac{\partial w}{\partial x}+\frac{u_{t}-u_{b}}{h_{c}}\right), \\
D_{f}=D_{t}+D_{b},
\end{gathered}
$$

where $D_{t}$ and $D_{b}$ represent the flexural rigidities of the top and bottom plates, respectively.
The longitudinal forces acting in the midplanes of each face plate, denoted by $P_{t}$ and $P_{b}$, are in equilibrium on the whole section so that $P_{t}=-P_{b}$, where

$$
P_{t}=A_{t} \frac{\partial u_{t}}{\partial x}, \quad P_{b}=A_{b} \frac{\partial u_{b}}{\partial x},
$$

where $A_{t}$ and $A_{b}$ represent the extensional rigidities of the top and bottom plates, respectively.

When no external loads are applied,

$$
\frac{\partial Q}{\partial x}=0
$$

From (3) and (5) we get the axial force as

$$
P_{t 1,3,5}=-P_{b 1,3,5}=\frac{D_{f}}{D_{g} d}\left(\frac{1}{b} \frac{\partial^{4} w}{\partial x^{4}}-D_{g} R \frac{\partial^{2} w}{\partial x^{2}}\right),
$$

where

$$
D_{g}=\frac{G_{c}}{h_{c}}\left(\frac{1}{A_{t}}+\frac{1}{A_{b}}\right), \quad R=\frac{d^{2}}{D_{f}}\left(\frac{A_{t} A_{b}}{A_{t}+A_{b}}\right) .
$$

The moment $M_{c}$ of the core is associated with equal and opposite longitudinal forces $P_{t}$ and $P_{b}$ acting along the midplanes of the face plates, as given by

$$
M_{c}=P_{b} d=\frac{-D_{f}}{D_{g}}\left(\frac{1}{b} \frac{\partial^{4} w}{\partial x^{4}}-D_{g} R \frac{\partial^{2} w}{\partial x^{2}}\right) .
$$

The total bending moment of the intact sandwich beam can be obtained as the sum of the moment in (9) and the moments for the faceplates:

$$
M_{i}=\frac{-D_{f}}{D_{g}}\left(\frac{1}{b} \frac{\partial^{4} w}{\partial x^{4}}-D_{g}(1+R) \frac{\partial^{2} w}{\partial x^{2}}\right), \quad i=1,3,5
$$


The total shear force $Q_{i}$ can be found as

$$
\begin{gathered}
Q_{i}=\frac{\partial M_{i}}{\partial x} \\
Q_{i}=\frac{-D_{f}}{D_{g}}\left(\frac{1}{b} \frac{\partial^{5} w}{\partial x^{5}}-D_{g}(1+R) \frac{\partial^{3} w}{\partial x^{3}}\right), \quad i=1,3,5 .
\end{gathered}
$$

Differentiating (12) and incorporating the inertia terms, the equation of motion for the flexural vibration of the intact sandwich beam can be obtained as

$$
\begin{array}{r}
\frac{1}{b} \frac{\partial^{6} w_{i}}{\partial x^{6}}-D_{g}(1+R) \frac{\partial^{4} w_{i}}{\partial x^{4}}+\frac{m}{D_{f}}\left(\frac{1}{b} \frac{\partial^{4} w_{i}}{\partial x^{2} \partial t^{2}}-D_{g} \frac{\partial^{2} w_{i}}{\partial t^{2}}\right)=0 \\
i=1,3,5
\end{array}
$$

3.2. Formulation for the Debonded Region of the Beam. For the debonded beam segment, the equation of motion according to Euler beam theory is

$$
\frac{\partial^{4} w_{i}}{\partial x^{4}}+\frac{m}{D_{f}} \frac{\partial^{2} w_{i}}{\partial t^{2}}=0, \quad \text { for } i=2,4
$$

The bending moment and shear force of $M_{2,4}$ and $Q_{2,4}$ on the section of the debonded beam are

$$
M_{2,4}=-D_{f} \frac{\partial^{2} w}{\partial x^{2}}, \quad Q_{2,4}=-D_{f} \frac{\partial^{3} w}{\partial x^{3}}
$$

3.3. Analytical Solution. Assuming free harmonic vibration, $w_{i}(x, t)=W_{i}(x) e^{i w t}$, and scaling $x$ such that $x=L \xi$, we can rewrite (13) as

$$
\frac{\partial^{6} W_{i}}{\partial \xi^{6}}-C_{1} \frac{\partial^{4} W_{i}}{\partial \xi^{4}}-C_{2} \frac{\partial^{2} W_{i}}{\partial \xi^{2}}+C_{3} W_{i}=0, \quad i=1,3,5,
$$

where

$$
C_{1}=D_{g} b L^{2}(1+R), \quad C_{2}=\frac{m \omega^{2} L^{4}}{D_{f}}, \quad C_{3}=C_{2} b D_{g} L^{2}
$$

The solution of (16) will lead to six roots of $\lambda_{j}$ for each region $i=1,3,5$ and the displacement function for each beam $i=$ $1,3,5$ can be written as

$$
\begin{aligned}
& W_{1}(\xi)=\sum_{j=1}^{6} A_{j} e^{\lambda_{j} \xi}, \\
& W_{3}(\xi)=\sum_{j=11}^{16} A_{j} e^{\lambda_{j} \xi}, \\
& W_{5}(\xi)=\sum_{j=21}^{26} A_{j} e^{\lambda_{j} \xi},
\end{aligned}
$$

where $A_{j}$ represents a set of six constants for each region $i=$ $1,3,5$.
Similarly, the solution of (14) results in four roots for $\lambda_{j}$ and for the beams $i=2$ and 4 :

$$
\begin{aligned}
& W_{2}(\xi)=\sum_{j=7}^{10} A_{j} e^{\lambda_{j} \xi}, \\
& W_{4}(\xi)=\sum_{j=17}^{20} A_{j} e^{\lambda_{j} \xi} .
\end{aligned}
$$

The general solutions for the entire debonded sandwich beam are given by (18) and (19). The twenty-six unknowns $A_{j},(j=1,2, \ldots, 26)$ and the frequency variable $\omega$ are determined using the appropriate boundary conditions of intact regions and continuity/equilibrium conditions at debond boundaries. The six end conditions and twenty compatibility/equilibrium conditions provide 26 homogenous equations for the unknowns $A_{j}, j=(1,2, \ldots, 26)$. The characteristic equation for the Eigen values is obtained by equating the determinant of the coefficient matrix to zero and the frequency variable $\omega$ is determined.

\section{Finite Element Analysis}

For the CFRP skinned honeycomb sandwich beam, the geometry of the honeycomb core is retained as it is and 2D shell element with six degrees of freedom per node is used to model the core. It is necessary to use $3 \mathrm{D}$ brick element (with three translational degrees of freedom per node) for top and bottom skins to incorporate through-width interface debond regions at predetermined symmetric locations more precisely. Contact elements are introduced between the elements of skin and core at the debond locations to prevent the debonded skin from overlapping with the core during vibration. The contact elements are capable of supporting only compression in the direction normal to the surfaces and have three translational degrees of freedom at each node. The finite element model is shown in Figure 3.

In the case of debonded beams, as the contact elements introduce nonlinearity, transient analysis (time-history analysis) is performed. Time-history analysis is a technique used to determine the dynamic response of a structure under the action of any general time-dependent loads. A constant impulse force $(F)$ is applied on the node at the edge of the beam and the displacement response curve is obtained for the same location as in Figure 4. Fast Fourier transform (FFT) analysis is performed on the results of time-history analysis to obtain the Frequency Response Function (FRF) curve as shown in Figure 5.

\section{Results and Discussion}

5.1. Single Debond at Top and Bottom Interface at Symmetric Locations. Study is carried out for eight cases of single debond with $L_{d 1}$ varying from $0.05 \mathrm{~L}$ to $0.40 \mathrm{~L}$ and the debond starts at $L_{1}=20 \mathrm{~mm}$ from the fixed end (Figure 1). Frequencies are evaluated for the cantilever CFRP skinned sandwich beam using present analytical approach and finite element analysis. Figure 6 gives a comparison of frequencies by both the methods. It was reported in author's earlier 


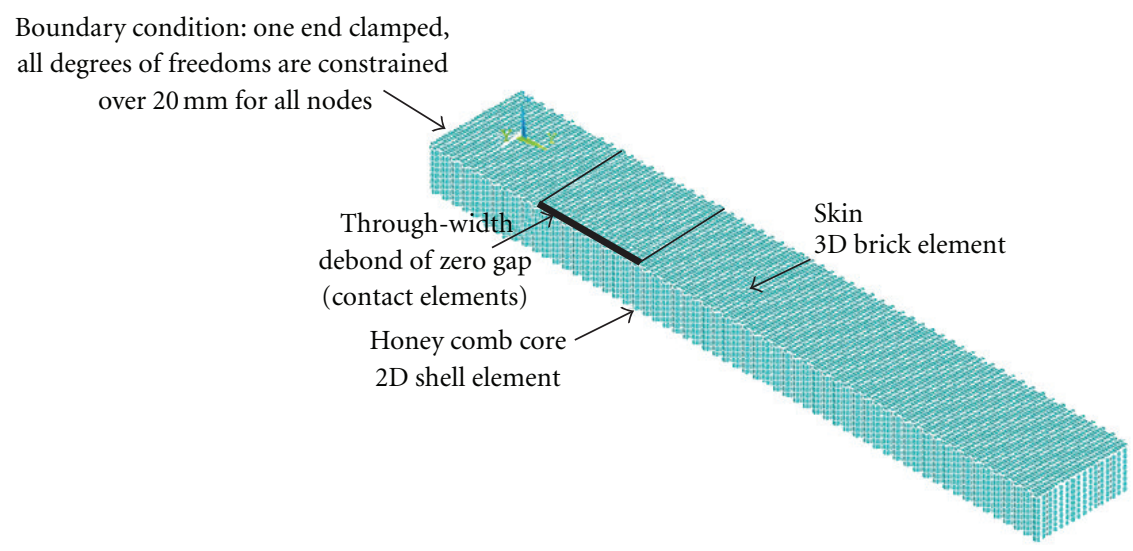

FIGURE 3: Finite element model of the honeycomb sandwich structure.

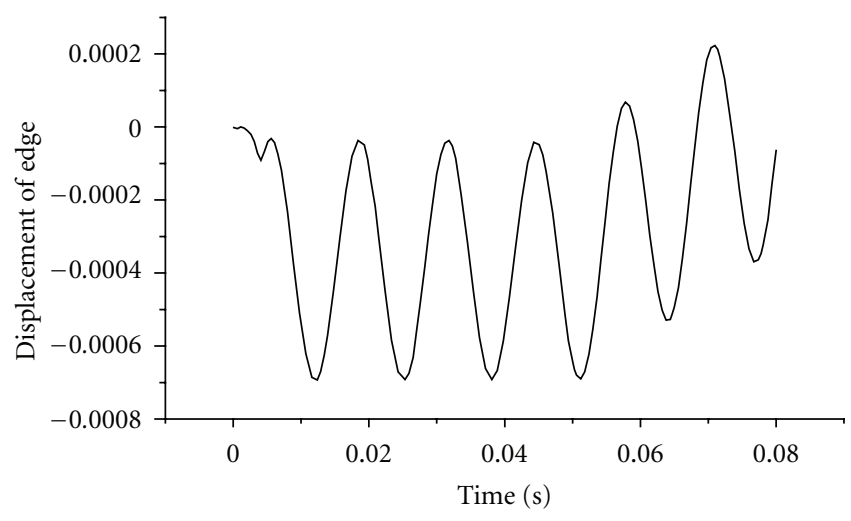

FIgURE 4: Time-displacement response curve.

Frequency $(\mathrm{Hz})$

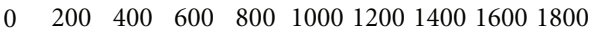

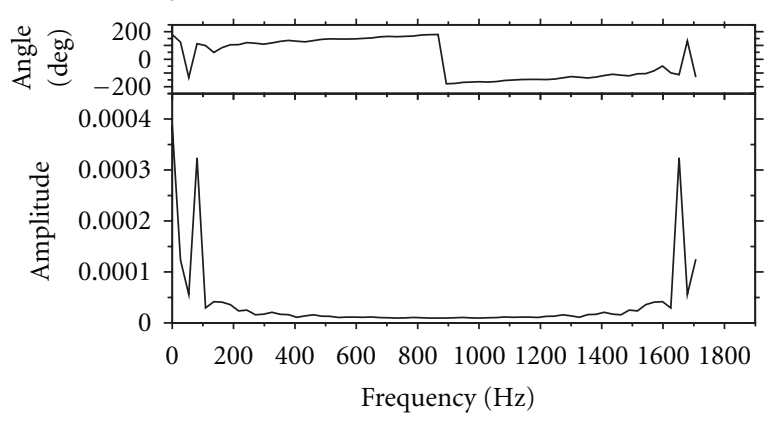

Figure 5: Frequency response curve.

work [1] that, for debond up to $12 \%$ of beam length, a good agreement between the test and analytical solution (based on the formulation of split beam theory including core stiffness) was obtained. In principle, analytical formulation underestimates the stiffness, as the contact between the honeycomb core and the skin during vibration as it occurs in the actual case could not be modeled. Hence, in the absence of more test data, finite element analysis with contact element in the debond region is used in the present work, and

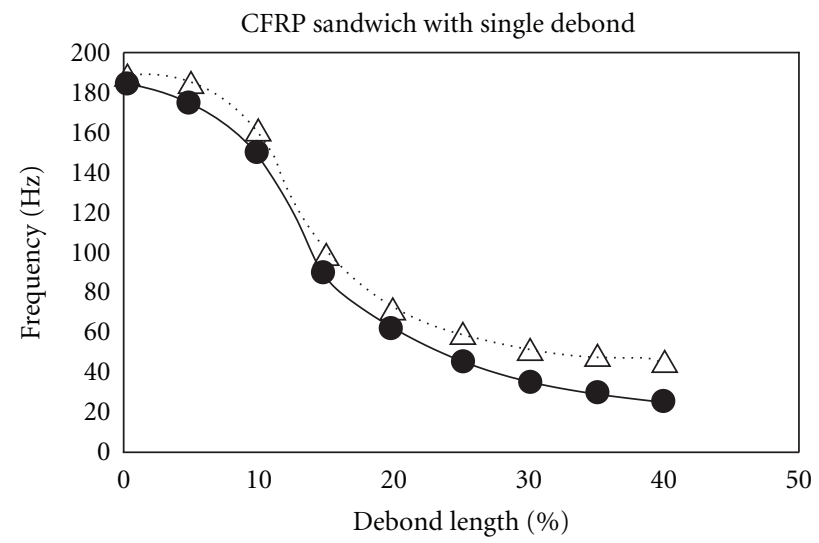

$\triangle$ FEM
- Analytical

FIGURE 6: Comparison of fundamental frequency of CFRP sandwich beam with single debond obtained by analysis and numerical approach.

good agreement is seen between the two results for debond up to $15 \%$ of beam length (Figure 6). Beyond this limit, for $20 \%$ debond length, the deviation between analytical solution and finite element method is $11 \%$ while for $40 \%$ debond length it is nearly $40 \%$. Hence, it can be concluded that for a single debond in a sandwich beam, the analytical solution is valid up to $15 \%$ of debond length.

For higher modes, a similar agreement in frequencies up to $15 \%$ of debond length is observed as in Figure 7.

\subsection{Multiple Debond at Top and Bottom Interface at Symmet-} ric Locations. Fundamental frequency obtained by analytical solution is compared with finite element analysis for CFRP sandwich beams with multiple debond and given in Table 2 . Two debonds of same size $(100 \mathrm{~mm})$ at different spacing (50 $\mathrm{mm}$ and $150 \mathrm{~mm}$, resp.) as in [1] are considered here and it is observed that the two results agree well. In other words, for $15 \%$ debond length each, even though the total defect 
TABLE 2: Comparison of fundamental frequency of cantilever CFRP sandwich beam with multiple debonds .

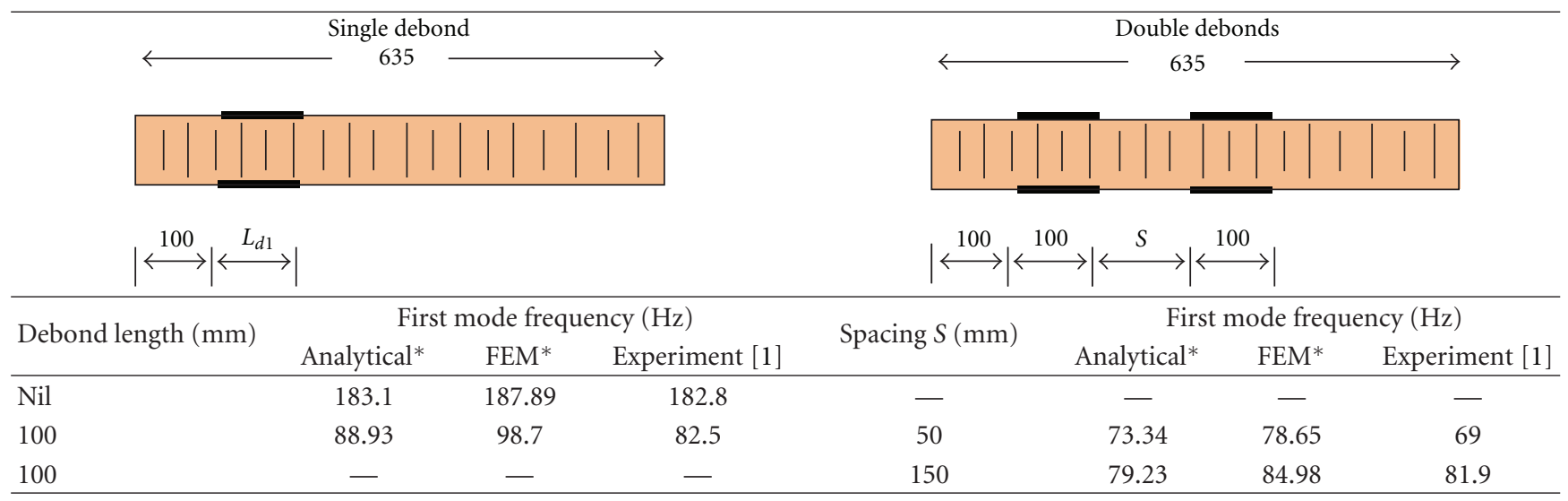

${ }^{*}$ Present results.

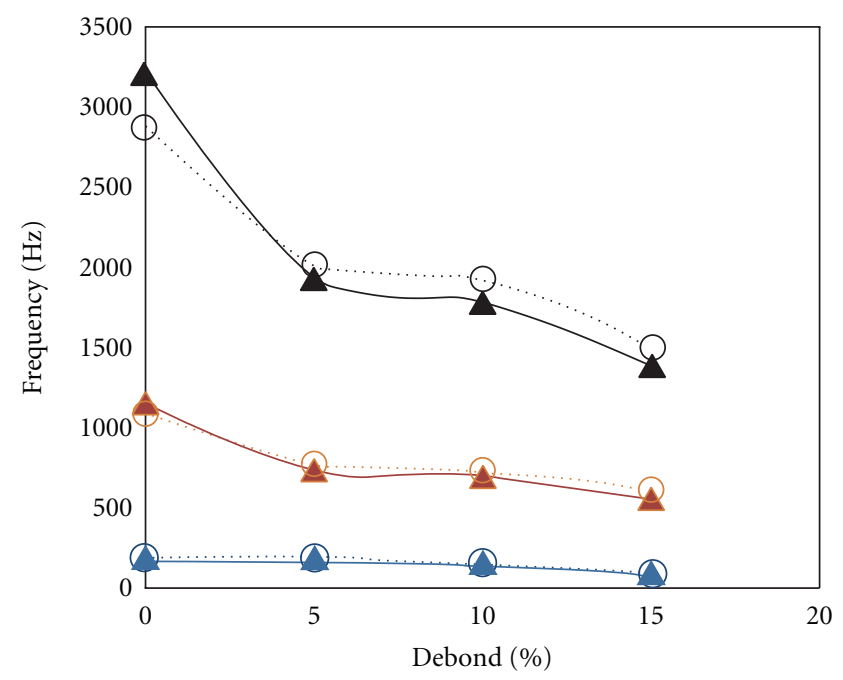
- Mode 1 analytical
$\bigcirc$. Mode 1 FEM
Mode 2 analytical
Mode 3 analytical
. Mode 2 FEM
$\bigcirc$. Mode 3 FEM

FIGURE 7: Comparison of higher mode frequencies of CFRP sandwich beam with single debond obtained by analysis and numerical approaches.

length is $30 \%$ of beam length, good agreement is obtained between the test, analysis, and finite element results.

Study is further extended for sandwich beam with two debonds at different spacing between them, keeping the first debond location same as mentioned earlier $\left(L_{1}=\right.$ $20 \mathrm{~mm}$ ). The variation of frequency as the spacing increases is depicted in Figure 8. It compares the frequencies evaluated by analytical method and finite element method. It may be noted that when two $0.05 \mathrm{~L}$ debonds are very close to each other (about 1.5 times single debond length), the reduction in frequency of the structure with respect to intact beam is observed as about 3\% and 5\% by finite element method and analytical solution, respectively. Similar deviations noted for a single debond of $0.10 \mathrm{~L}(0.05 \mathrm{~L}+.05 \mathrm{~L})$ are about $14 \%$ and $18 \%$, respectively. It implies that a single long debond

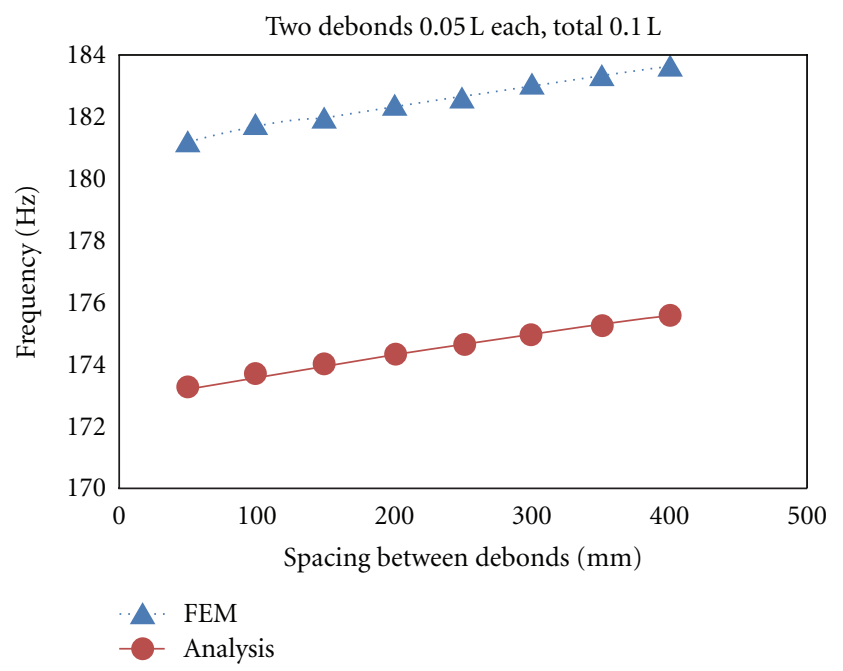

(a)

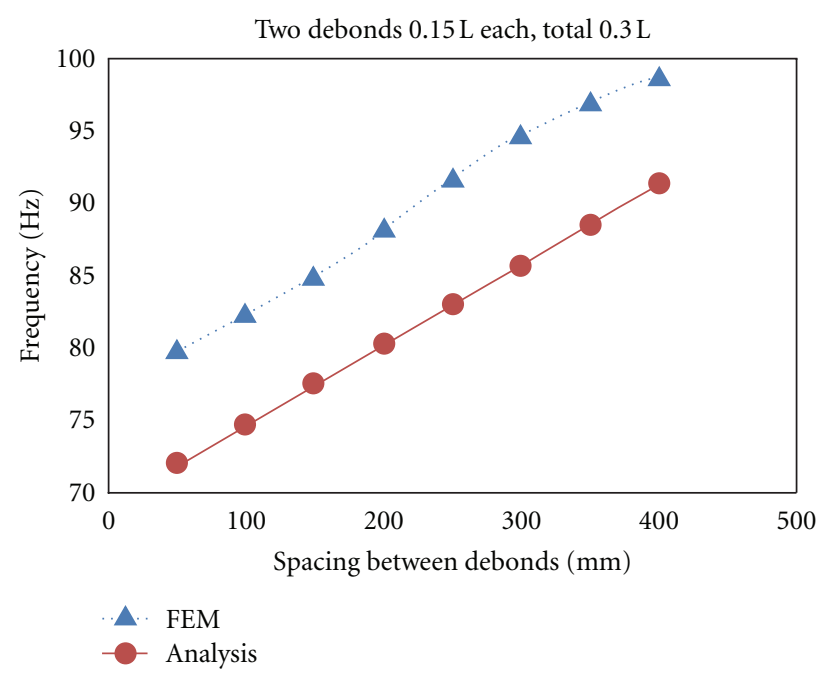

(b)

Figure 8: Variation in frequency with respect to spacing between debonds. 
is more significant than multiple debonds of comparable length located in the axial direction of beam.

It can be concluded that, in the case of double debonds, even though total debond length is $30 \%$ of beam length, when they are well separated (by about three times the single debond length), frequency of the sandwich beam is reduced only as much as in the presence of single debond.

\section{Conclusions}

Analytical formulation for the evaluation of frequency of CFRP sandwich beam with debonds, using the split beam theory, has been carried out considering honeycomb core stiffness and validated the range of debond size through 3D nonlinear transient analysis. Honeycomb core is modeled with the contact element introduced between the core and the skin during vibration, following fast Fourier transform analysis to obtain the Frequency Response Functions. Comparison of fundamental frequencies of a cantilever beam with a single debond obtained by the test, analysis, and numerical method show a reasonably good agreement between them up to a debond length of $15 \%$ of beam length. Analytical model has also been validated for the case of double debonds and it is observed that reduction in frequency of the sandwich beam is only as much as with the presence of single debond when two debonds (of sum total length up to $30 \%$ of beam length) are separated by three times single-debond size. However, when the double-debond size is very small up to a value of $5 \%$ of the beam length, then the distance in between can be retained to 1.5 time the debond size to obtain a value corresponding to a single debond.

\section{References}

[1] B. Saraswathy, L. Mangal, and R. Ramesh Kumar, "Analytical approach for model characteristics of honeycomb sandwich beams with multiple debond," Journal of Sandwich Structures and Materials. In press.

[2] S. Goswami and W. Becker, "Analysis of debonding fracture in a sandwich plate with hexagonal core," Composite Structures, vol. 49, no. 4, pp. 385-392, 2000.

[3] K. Waldron, A. Ghoshal, M. J. Schulz et al., "Damage detection using finite element and laser operational deflection shapes," Finite Elements in Analysis and Design, vol. 38, no. 3, pp. 193226, 2002.

[4] H. S. Kim, A. Chattopadhyay, and A. Ghoshal, "Characterization of delamination effect on composite laminates using a new generalized layerwise approach," Computers and Structures, vol. 81, no. 15, pp. 1555-1566, 2003.

[5] L. H. Yam, Z. Wei, L. Cheng, and W. O. Wong, "Numerical analysis of multi-layer composite plates with internal delamination," Computers and Structures, vol. 82, no. 7-8, pp. 627-637, 2004.

[6] A. Cecchini, "Damage detection and identification in sandwich composites using neural networks," International Journal of Solids and Structures, vol. 50, pp. 621-700, 2005.

[7] W. Lestari and P. Qiao, "Dynamic characteristics and effective stiffness properties of honeycomb composite sandwich structures for highway bridge applications," Journal of Composites for Construction, vol. 10, no. 2, pp. 148-160, 2006.
[8] V. N. Burlayenko and T. Sadowski, "Analysis of structural performance of sandwich plates with foam-filled aluminum hexagonal honeycomb core," Computational Materials Science, vol. 45, no. 3, pp. 658-662, 2009.

[9] V. N. Burlayenko and T. Sadowski, "Influence of skin/core debonding on free vibration behavior of foam and honeycomb cored sandwich plates," International Journal of Non-Linear Mechanics, vol. 45, no. 10, pp. 959-968, 2010. 

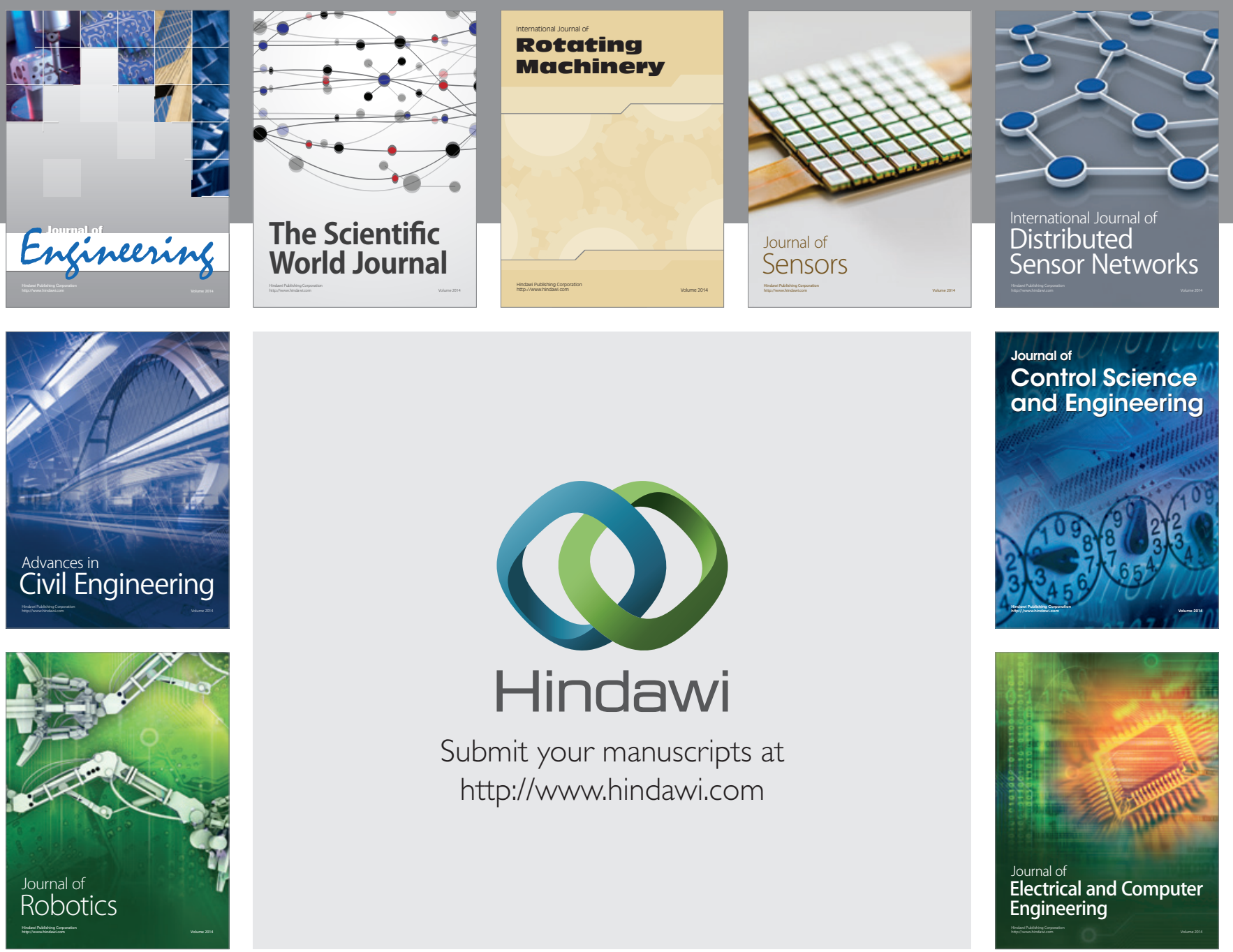

Submit your manuscripts at

http://www.hindawi.com
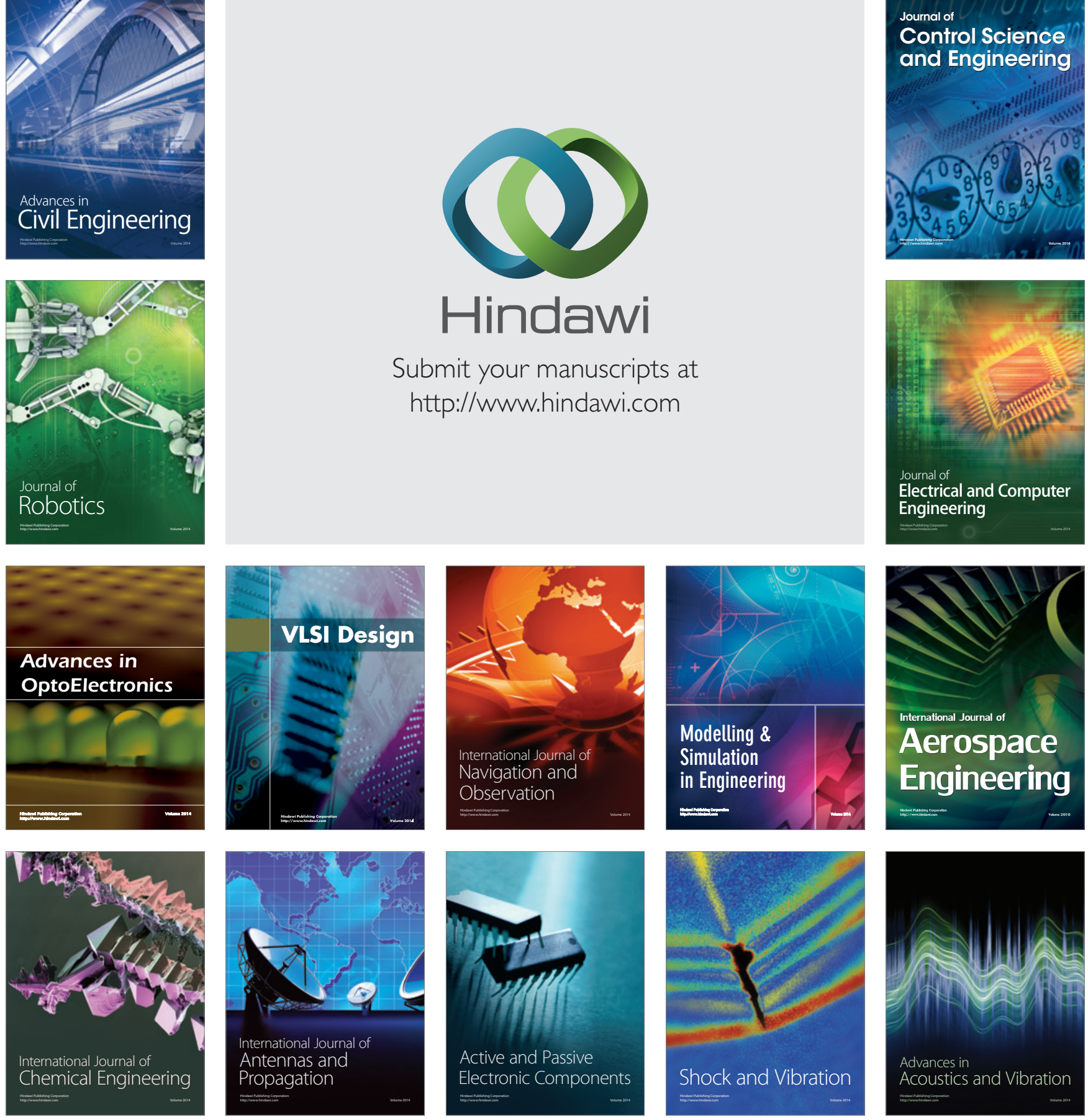